\title{
Very low scale Coleman-Weinberg inflation with nonminimal coupling
}

\author{
Kunio Kaneta, ${ }^{1}$ Osamu Seto, ${ }^{2,3}$ and Ryo Takahashi ${ }^{4}$ \\ ${ }^{1}$ Center for Theoretical Physics of the Universe, Institute for Basic Science (IBS), Daejeon 34051, Korea \\ ${ }^{2}$ Institute for International Collaboration, Hokkaido University, Sapporo 060-0815, Japan \\ ${ }^{3}$ Department of Physics, Hokkaido University, Sapporo 060-0810, Japan \\ ${ }^{4}$ Graduate School of Science, Tohoku University, Sendai 980-8578, Japan
}

(Received 10 October 2017; published 7 March 2018)

\begin{abstract}
We study viable small-field Coleman-Weinberg (CW) inflation models with the help of nonminimal coupling to gravity. The simplest small-field CW inflation model (with a low-scale potential minimum) is incompatible with the cosmological constraint on the scalar spectral index. However, there are possibilities to make the model realistic. First, we revisit the CW inflation model supplemented with a linear potential term. We next consider the $\mathrm{CW}$ inflation model with a logarithmic nonminimal coupling and illustrate that the model can open a new viable parameter space that includes the model with a linear potential term. We also show parameter spaces where the Hubble scale during the inflation can be as small as $10^{-4} \mathrm{GeV}$, $1 \mathrm{GeV}, 10^{4} \mathrm{GeV}$, and $10^{8} \mathrm{GeV}$ for the number of $e$-folds of 40,45 , 50, and 55, respectively, with other cosmological constraints being satisfied.
\end{abstract}

DOI: 10.1103/PhysRevD.97.063004

\section{INTRODUCTION}

Inflation is one of the successful paradigms in modern cosmology that can address various cosmological issues [1-3] and generate primordial perturbations [4-7]. The underlying particle physics is, however, still unclear, and it is indispensable in understanding physics in the early Universe. To this end, in particular, it is legitimate to ask what is a consistent inflationary scenario for a specific physics model beyond the standard model of particle physics.

Two categories are often used to classify various inflationary models: large-field and small-field inflation, according to whether the inflaton field excursion during inflation exceeds the Planck scale or not. Each class of models has its own virtues. For instance, the large-field models have an advantage in the initial condition of inflation [8,9], whereas in the small-field models, inflation can take place with the inflaton field value well below the Planck scale, and hence, its field theoretical description is verified and well understood.

In small-field models with a symmetry-breaking-type potential, inflation takes place at the vicinity of the origin, and the inflaton field slowly rolls down toward the potential minimum located below the Planck scale. From the

Published by the American Physical Society under the terms of the Creative Commons Attribution 4.0 International license. Further distribution of this work must maintain attribution to the author(s) and the published article's title, journal citation, and DOI. Funded by SCOAP. normalization of temperature anisotropy of cosmic microwave background $(\mathrm{CMB})$ radiation of $\mathcal{O}\left(10^{-5}\right)$, the energy scale of small-field inflation models, which is equivalently the Hubble parameter during inflation, turns out to be rather small. Thus, a small-field inflation generally leads to a rather low reheating temperature.

Such a low-scale inflation and its resultant low reheating temperature are attractive from several viewpoints. Here, we note several examples and those motivations. First, in a Peccei-Quinn (PQ) extended model to solve the strong $C P$ problem in the standard model of particle physics [10,11], if the PQ symmetry is broken before or during inflation, axion fluctuations on the order of the Hubble parameter during inflation are generated and induce axion isocurvature perturbations [12-15]. To satisfy the stringent bound on axion isocurvature perturbation by the $\mathrm{CMB}$ temperature anisotropy, a small Hubble parameter during inflation, $\lesssim 10^{7} \mathrm{GeV}$, is required [16]. Second, one of the most promising scenarios for generation of baryon asymmetry is the Affleck-Dine mechanism with a flat direction [17]. An appropriate amount of baryon asymmetry can be generated by a flat direction lifted by a dimension-six operator for a low reheating temperature $T_{R}$ of about $100 \mathrm{GeV}$ [18]. Affleck-Dine baryogenesis by such flat directions is interesting because it provides a solution to the coincidence of energy densities between baryon and dark matter with the formation of $Q$-balls [19-21]. Third, an issue of supersymmetric models in cosmology is the overproduction of the gravitino [22-24]. Because gravitino abundance produced through thermal scatterings is proportional to the reheating temperature after inflation, in order to avoid 
overproduction, the upper bound on the reheating temperature is imposed. For a recent estimation, see, e.g., Refs. [25,26]. Finally, the recently proposed relaxion mechanism [27], as a solution to the hierarchy problem of Higgs boson by utilizing a slowly rolling scalar field in the context of inflationary cosmology, also requires a very long period and a very low energy scale of inflation for a phase transition by QCD(-like) strong dynamics to take place during inflation, not only in the minimal model [27-29] but also in some extended models [30-33]. (However, for other extensions where the relaxion mechanism can work at relatively high scale, see, e.g., Refs. [34-36].)

In this paper, we pursue a possible realization of viable small-field inflationary models based on the ColemanWeinberg (CW) model [37]. In particular, we discuss how small inflation scale can be achieved in the $\mathrm{CW}$ model with some possible modifications. The $\mathrm{CW}$ inflation model is a typical model of low-scale and small-field inflation [38-40]. However, the original CW inflation model is doomed by the observed scalar spectral index [41], which is significantly larger than that of the model predictions. ${ }^{1}$ Iso et al. have proposed simple extensions to ameliorate this discrepancy [49]. In this paper, we revisit known examples of such extension and explore further possibilities by considering other promising extensions.

The rest of this paper is organized as follows. In Sec. II, we first go over the models discussed in Ref. [49] and move onto other possible extensions based on a nonminimal coupling to gravity. We devote Sec. III to discussions and conclusions.

\section{SMALL-FIELD COLEMAN-WEINBERG INFLATION MODEL}

We study a class of small-field CW inflation where the inflaton starts to roll down from the vicinity of the origin to the potential minimum [38-40,49]. The scalar potential for the inflaton $\phi$ is given by

$V(\phi)=\frac{A}{4} \phi^{4}\left(\ln \frac{\phi^{2}}{M^{2}}-\frac{1}{2}\right)+V_{0}, \quad V_{0}=\frac{A M^{4}}{8}$,

with a scale $M\left(<M_{\mathrm{pl}}\right)$ and $M_{\mathrm{pl}}$ being the reduced Planck mass. $V_{0}$ is determined by the vanishing cosmological constant at the minimum. Derivatives of the potential with respect to $\phi$ are

$$
V^{\prime}=A \phi^{3} \ln \frac{\phi^{2}}{M^{2}}, \quad V^{\prime \prime}=A \phi^{2}\left(2+3 \ln \frac{\phi^{2}}{M^{2}}\right) .
$$

\footnotetext{
${ }^{1}$ If the vacuum expectation value of the field is larger than the Planck scale, the $\mathrm{CW}$ potential might reproduce the consistent density perturbation [42] (for other attempts, see, e.g., [43-48]). However, this case belongs to the large-field model and is not discussed in this paper.
}

We find that the vacuum expectation value at the minimum is given by $\langle\phi\rangle=M$, and $V_{0}$ is obtained by $V(M)=0$ as shown above. Thus, the slow roll parameters are calculated as

$$
\begin{gathered}
\epsilon=\frac{M_{\mathrm{pl}}^{2}}{2}\left(\frac{V^{\prime}}{V}\right)^{2} \simeq 32\left(\frac{M_{\mathrm{pl}}}{M}\right)^{2}\left(\frac{\phi}{M}\right)^{6}\left(\ln \frac{\phi^{2}}{M^{2}}\right)^{2}, \\
\eta=M_{\mathrm{pl}}^{2}\left(\frac{V^{\prime \prime}}{V}\right) \simeq 24\left(\frac{M_{\mathrm{pl}}}{M}\right)^{2}\left(\frac{\phi}{M}\right)^{2} \ln \frac{\phi^{2}}{M^{2}},
\end{gathered}
$$

where $V \simeq V_{0}$ is utilized in a small-field region of $\phi$. The slow roll conditions can be satisfied when a field value of $\phi$ is small enough to satisfy $|\eta|<1$ for a given $M$. In this region, we find $\epsilon \ll|\eta|$ and $\phi \ll M$. This simple CW inflation model leads to a tiny tensor-to-scalar ratio $(r \simeq 16 \epsilon)$, which is allowed by the current bound $[16,50]$ from cosmological observations. However, for $M \ll M_{\mathrm{p} \text { l }}$, the scalar spectral index $\left(n_{s} \simeq 1+2 \eta-6 \epsilon\right)$ from this model as $0.94 \lesssim n_{s} \lesssim$ 0.95 for $50 \leq N_{*} \leq 60$ does not fall into the allowed region for a tiny $r$ as

$$
\begin{aligned}
& 0.955 \lesssim n_{s} \lesssim 0.976 \quad(68 \% \mathrm{CL}), \\
& 0.949 \lesssim n_{s} \lesssim 0.982 \quad(95 \% \mathrm{CL}),
\end{aligned}
$$

given by Planck TT + low $P$ data [16]. $N_{*}$ is the number of $e$-folds given by

$N_{*}=\frac{1}{M_{\mathrm{pl}}^{2}} \int_{\phi_{\mathrm{end}}}^{\phi_{*}} \frac{V}{V^{\prime}} d \phi \simeq \frac{1}{M_{\mathrm{pl}}^{2}} \int_{\phi_{\mathrm{end}}}^{\phi_{*}} \frac{V_{0}}{A \phi^{3} \ln \left(\phi^{2} / M^{2}\right)} d \phi$,

with field value $\phi_{*}$ where the pivot scale $k_{*}$ exits from the Hubble radius. $\phi_{\text {end }}$ denotes the field value at the end of inflation. Therefore, the low-scale CW inflation model must be modified to be consistent with cosmological observations. In most studies, $N_{*}$ is taken to be about 50 or 60 . In fact, $N_{*}$ weakly depends on the energy scale of inflation and the delay of reheating after inflation [51-53] as

$$
N_{*} \simeq 62-\ln \frac{10^{16} \mathrm{GeV}}{V_{*}^{1 / 4}}-\frac{1}{3} \ln \frac{V_{*}^{1 / 4}}{\rho_{R}^{1 / 4}},
$$

with the energy density at the reheating $\rho_{R}$ and the energy density at the moment of the pivot scale horizon crossing during inflation $V_{*}$ for the standard thermal history after inflation in which the Universe becomes the matter dominated with the equation of state $w=0$ during the coherent oscillation of inflaton after inflation, followed by the radiationdominated Universe. Here, we used $V_{*} \simeq V\left(\phi_{\text {end }}\right)$. Now, $N_{*}$ is a function of $V_{*}$ and $\rho_{R}$. In the following analysis, because we are interested in very low scale $\mathrm{CW}$ inflation, we vary $N_{*}$ from 40 to 55 under the condition $\rho_{R} \leq V_{*}$.

\section{A. Fermion condensates}

A possibility to increase $n_{s}$ is the introduction of a linear term, which can be generated by a fermion condensation in 
the inflaton potential discussed in Ref. [49]. In the work, two examples that induce a linear term have been shown. One is the condensation of right-handed neutrinos $N$, which couple to $\phi$ through a Yukawa interaction $y_{N} \phi \bar{N}^{c} N$. The other one is the chiral condensation, which generates a linear term as $C_{h} h$ in the Higgs $(h)$ potential. Then, the mixing between the Higgs and inflaton induces a linear term in the inflaton potential. In both cases, a linear term, $C \phi$, in the inflaton potential can be induced from a fermion condensate.

The potential (1) is changed to

$V(\phi)=\frac{A}{4} \phi^{4}\left(\ln \frac{\phi^{2}}{M^{2}}-\frac{1}{2}\right)-C \phi+V_{0}, \quad V_{0}=\frac{A M^{4}}{8}$.

$V^{\prime}$ and $\epsilon$ are also modified to

$$
\begin{aligned}
V^{\prime} & =A \phi^{3} \ln \frac{\phi^{2}}{M^{2}}-C, \\
\epsilon & \simeq 32\left(\frac{M_{\mathrm{pl}}}{M}\right)^{2}\left[\left(\frac{\phi}{M}\right)^{3} \ln \frac{\phi^{2}}{M^{2}}-\frac{C}{A M^{3}}\right]^{2} .
\end{aligned}
$$

$V^{\prime \prime}$ is unchanged, but $N_{*}$ is modified as

$$
N_{*} \simeq \frac{1}{M_{\mathrm{pl}}^{2}} \int_{\phi_{\mathrm{end}}}^{\phi_{*}} \frac{V_{0}}{A \phi^{3} \ln \left(\phi^{2} / M^{2}\right)-C} d \phi .
$$

Thus, the relation between $n_{s}$ and $N_{*}$ changes from the original $\mathrm{CW}$ inflation.

For all figures in this paper, we normalize the amplitude of curvature perturbation at the pivot scale as $A_{s}=2.196 \times 10^{-9}$. Figure 1 shows $n_{s}$ as a function of $M$ in the model with fermion condensates. In the figure, black, dark gray, gray, and light gray curves correspond to $N_{*}=55,50,45$, and 40 cases, respectively. Solid and dashed curves are cases of $\tilde{C}=10^{-5}$ and $10^{-6}$, respectively, where $\tilde{C}$ is a dimensionless parameter defined as $\tilde{C} \equiv C\left(M_{\mathrm{pl}} / M\right)^{3} /\left(A M^{3}\right)$. The end of each curve corresponds to the case of the instantaneous reheating, $\rho_{R}=V_{*}$, which gives the maximal reheating temperature after inflation. The horizontal solid line denotes the center value of $n_{s}$ from the cosmological observation [16] as $n_{s}=0.9655$; its $1 \sigma$ range (5) is indicated by dashed lines, and shaded regions are outside of the bound (6). We find that the model has viable parameter space consistent with cosmological observations for $\tilde{C}=\mathcal{O}\left(10^{-5}\right)$, with $40 \lesssim$ $N_{*} \lesssim 55$ in the broad region of $M \gtrsim 10^{11} \mathrm{GeV}$. The additional contribution of $C$ in the denominator of Eq. (11) changes the value of $N_{*}$, but it does not change the magnitude of $\epsilon$, and thus the tensor-to-scalar ratio remains tiny as in the case of the original $\mathrm{CW}$ inflation model.

Next, we discuss the inflation scale, that is, the Hubble scale during the inflation, and maximal value of the number of $e$-folds during slow roll phase $N_{\max }$. The Hubble scale during the inflation $H_{\text {inf }}$ is approximated as

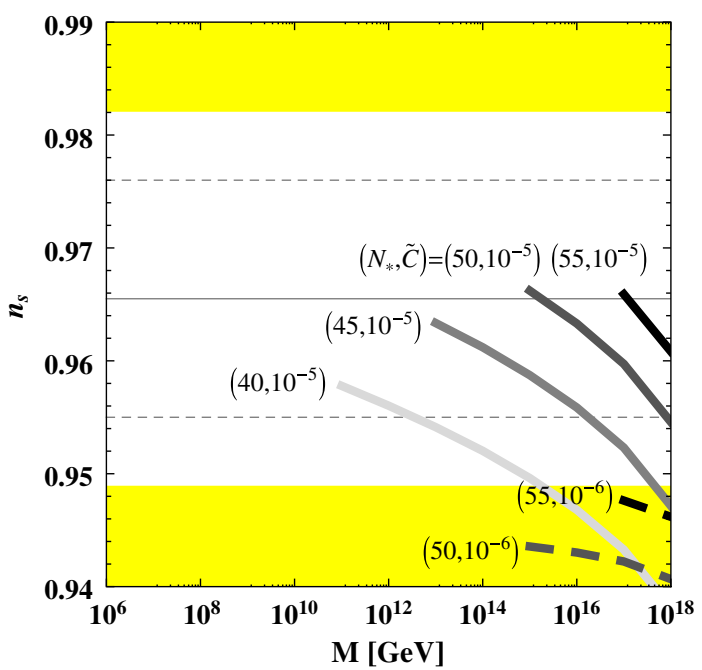

FIG. 1. Value of $n_{s}$ in the $\mathrm{CW}$ model with fermion condensates. Black, dark gray, gray, and light gray curves correspond to $N_{*}=55,50,45$, and 40 cases, respectively. Solid and dashed curves are $\tilde{C}=10^{-5}$ and $10^{-6}$, respectively, where $\tilde{C}$ is a dimensionless parameter defined as $\tilde{C} \equiv C\left(M_{\mathrm{pl}} / M\right)^{3} /\left(A M^{3}\right)$. The center values and $68 \% \mathrm{CL}$ and $95 \% \mathrm{CL}$ range of $n_{s}$ are shown by solid and dashed lines and shaded region, respectively.

$H_{\mathrm{inf}} \simeq \sqrt{V_{0} / 3 M_{\mathrm{pl}}^{2}}$ in the model. $N_{\mathrm{max}}$ could be an interesting quantity from the viewpoint of the relaxion scenario as stated in the Introduction. $N_{\max }$ is defined as

$$
N_{\max } \equiv \int_{\phi_{\text {end }}}^{\phi=0} \frac{V}{V^{\prime}} d \phi
$$

in this class of small-field inflation model. The values in this model are shown in Fig. 2. Solid, long dashed lines, and dashed curves in the right panel of Fig. 2 are $\tilde{C}=10^{-5}$, $10^{-5.5}$, and $10^{-6}$, respectively. Curves and regions in the figure are the same as those in Fig. 1. We find that possible inflation scale is $H_{\text {inf }} \gtrsim 10^{-4} \mathrm{GeV}, 1 \mathrm{GeV}, 10^{4} \mathrm{GeV}$, and $10^{8} \mathrm{GeV}$ for $N_{*}=40,45,50$, and 55 for $\tilde{C}=10^{-5}$, respectively. $M$ as large as $10^{18} \mathrm{GeV}$ is available for $A \sim \mathcal{O}\left(10^{-14}\right)$, and $H_{\text {inf }}$ can be reduced to be as low as $10^{-4} \mathrm{GeV}$ at $M=10^{11} \mathrm{GeV}$. Interestingly, this type of model can also realize small inflation scale compared with the usual large-field inflation models. The maximal value of the number of $e$-folds is $N_{\max } \simeq 110,140,240$, and 310 for $N_{*}=40,45,50$, and 55, respectively, with an appropriate value of $\tilde{C}$. Thus, enormous number of $e$-folds, which may be preferred in some relaxion models, cannot be realized in the model due to the absence of an extremely flat region such as a stationary point.

\section{B. Nonminimal coupling to gravity}

Let us now discuss another possible realization of a viable small-scale $\mathrm{CW}$ inflation, where we introduce a 

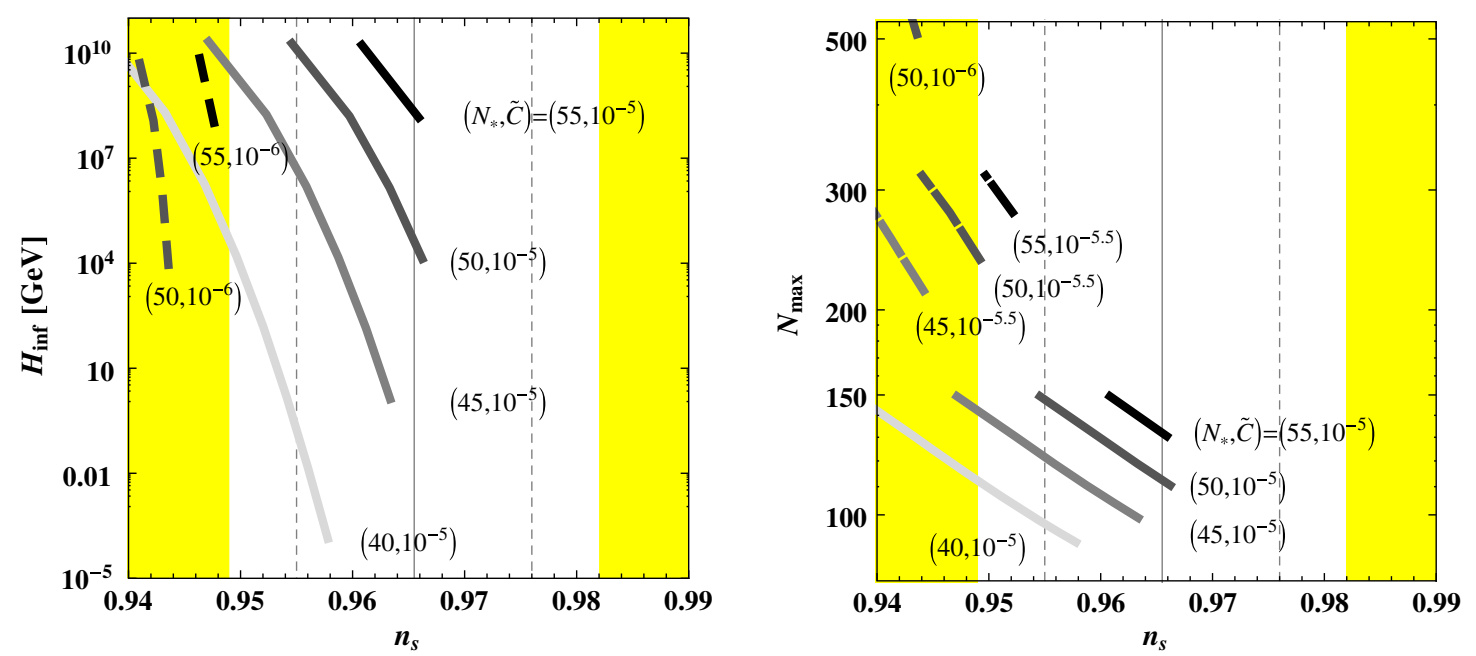

FIG. 2. Inflation scale (left) and maximal value of the number of $e$-folds (right) in this model. Solid, long dashed line, and dashed curves on the right are $\tilde{C}=10^{-5}, 10^{-5.5}$, and $10^{-6}$, respectively. (On the right, a dashed curve only appears in the upper-left corner.) The other meanings of curves and region in the figure are the same as those in Fig. 1.

nonminimal coupling of the inflaton to gravity. In Ref. [49], a nonminimal coupling to gravity of $\mathcal{L}_{\xi}=-\xi \bar{\phi}^{2} \mathcal{R} / 2$, with $\mathcal{R}$ being the Ricci scalar and $\bar{\phi}$ being the Jordan frame inflaton field, has been discussed, but it was concluded that this term cannot make the original $\mathrm{CW}$ inflation viable. ${ }^{2}$

Instead of utilizing the quadratic coupling to gravity, we introduce a logarithmic term of nonminimal coupling to gravity. Such a form of the nonminimal coupling may be obtained by incorporating quantum corrections to the $\bar{\phi}^{2} \mathcal{R}$ term [54], ${ }^{3}$ and here, we parametrize it as

$$
\mathcal{L}=-\xi M \bar{\phi} \mathcal{R}\left(\ln \frac{\bar{\phi}}{M}-c\right),
$$

in the Jordan frame. In this case, the potential (1) is changed to

$$
\begin{aligned}
V(\bar{\phi}) \rightarrow V_{E}(\bar{\phi}(\phi))= & \frac{V(\bar{\phi}(\phi))}{\Omega^{4}(\bar{\phi}(\phi))} \simeq \frac{A}{4} \bar{\phi}^{4}\left(\ln \frac{\bar{\phi}^{2}}{M^{2}}-\frac{1}{2}\right) \\
& +V_{0}\left(1+\frac{2 \xi M \bar{\phi}}{M_{\mathrm{pl}}^{2}}\left(\ln \frac{\bar{\phi}}{M}-c\right)\right),
\end{aligned}
$$

in the Einstein frame where $\Omega^{2} \equiv 1-\frac{\xi M \bar{\phi}}{M_{\mathrm{pl}}^{2}} \ln (\bar{\phi} / M-c)$, and $\phi$ is a canonically normalized inflaton field in this frame. Although one can safely approximate $\Omega^{2} \simeq 1$ and $d \phi / d \bar{\phi} \simeq$ 1 with $V(\bar{\phi}) \simeq V_{E}(\bar{\phi}(\phi)) \simeq V_{0}$ in a small-field region, the

\footnotetext{
${ }^{2}$ Furthermore, we have also checked that introductions of cubic $\left(\xi \bar{\phi}^{3} \mathcal{R} / M\right)$ and quartic $\left(\xi \bar{\phi}^{4} \mathcal{R} / M^{2}\right)$ terms in the original $\mathrm{CW}$ inflaton potential do not work. As $\bar{\phi}$ is taken as small values during the inflation, higher terms than the quadratic one do not drastically change the properties of the original $\mathrm{CW}$ inflation model.

${ }^{3}$ See also $[55-60]$.
}

additional term induced from the logarithmic form of nonminimal coupling to gravity cannot be negligible for $V_{E}^{\prime}$ and $V_{E}^{\prime \prime}$ in a certain parameter space. In particular, the model with a larger $c$ gives similar predictions from the $\mathrm{CW}$ model, with a linear term discussed in the previous subsection.

Taking derivatives with respect to $\phi$, we have

$$
V_{E}^{\prime}(\bar{\phi}(\phi))=\frac{d V_{E}}{d \bar{\phi}} \frac{1}{d \bar{\phi}} \simeq A \bar{\phi}^{3} \ln \frac{\bar{\phi}^{2}}{M^{2}}+\frac{A \xi M^{5}}{4 M_{\mathrm{pl}}^{2}}\left(\ln \frac{\bar{\phi}}{M}-c+1\right)
$$

$$
V_{E}^{\prime \prime}(\bar{\phi}(\phi)) \simeq A \bar{\phi}^{2}\left(2+3 \ln \frac{\bar{\phi}^{2}}{M^{2}}\right)+\frac{A \xi M^{5}}{4 M_{\mathrm{pl}}^{2} \bar{\phi}}
$$

Then, the slow roll parameters are calculated as

$$
\begin{array}{r}
\epsilon=\frac{M_{\mathrm{pl}}^{2}}{2}\left(\frac{V_{E}^{\prime}}{V_{E}}\right)^{2} \simeq 32\left(\frac{M_{\mathrm{pl}}}{M}\right)^{2} \\
\times\left[\left(\frac{\phi}{M}\right)^{3} \ln \frac{\phi^{2}}{M^{2}}+\frac{\xi}{4}\left(\frac{M}{M_{\mathrm{pl}}}\right)^{2}\left(\ln \frac{\phi}{M}-c+1\right)\right]^{2} \\
\eta=M_{\mathrm{pl}}^{2}\left(\frac{V_{E}^{\prime \prime}}{V_{E}}\right) \simeq 24\left(\frac{M_{\mathrm{pl}}}{M}\right)^{2} \\
\times\left[\left(\frac{\phi}{M}\right)^{2} \ln \frac{\phi^{2}}{M^{2}}+\frac{\xi}{12}\left(\frac{M}{M_{\mathrm{pl}}}\right)^{2}\left(\frac{M}{\phi}\right)\right],
\end{array}
$$

where $V_{E} \simeq V_{0}$ is utilized in the small-field region. $N_{*}$ becomes 

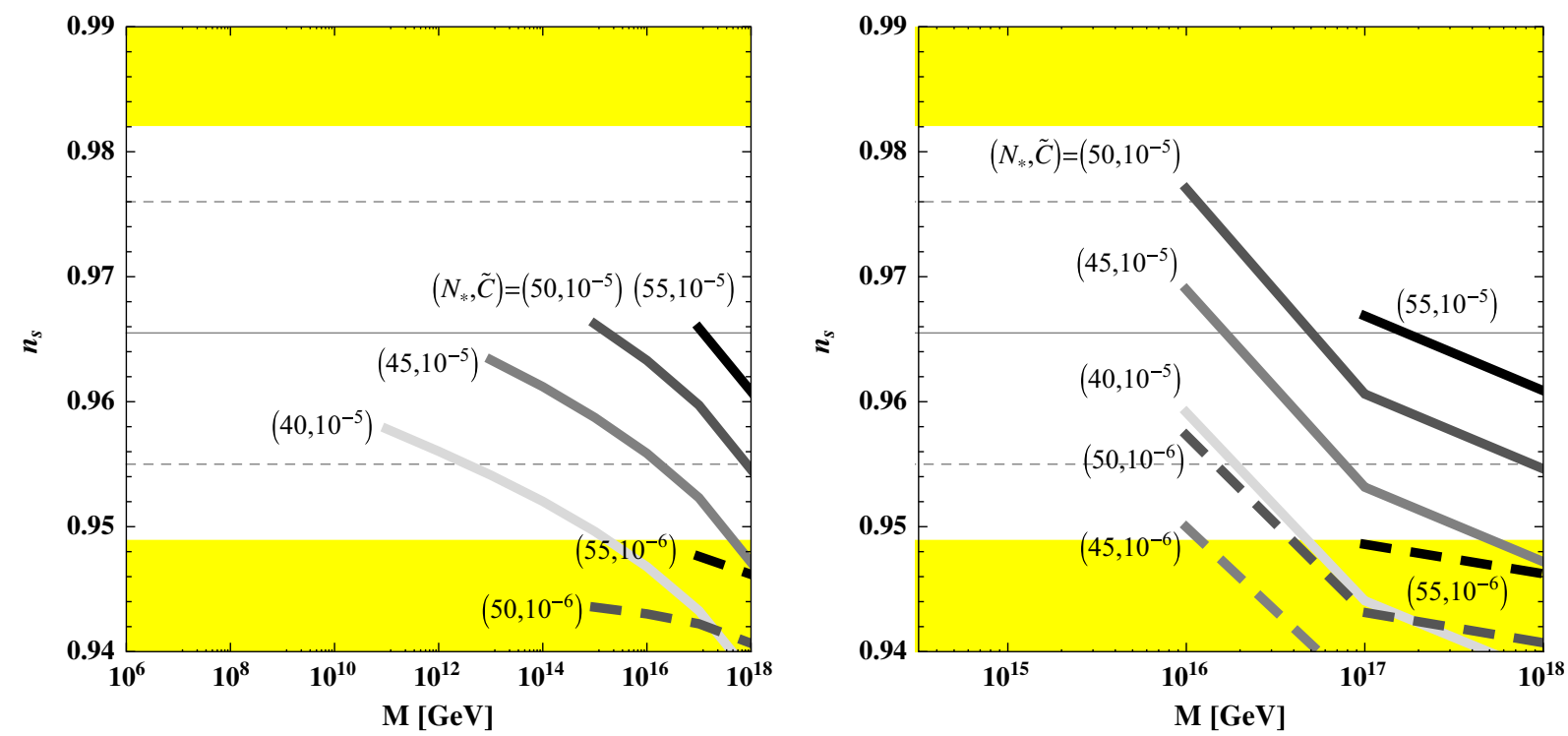

FIG. 3. Value of $n_{s}$ in the $\mathrm{CW}$ model with a logarithmic form of nonminimal coupling to gravity. Left and right panels correspond to $\xi=10^{-16}$ and $10^{-8}$, respectively. The meanings of curves and region in the figures are the same as those in Fig. 1.

$N_{*} \simeq \frac{1}{M_{\mathrm{pl}}^{2}} \int_{\phi_{\mathrm{end}}}^{\phi_{*}} \frac{V_{0}}{\left[A \bar{\phi}^{3} \ln \left(\bar{\phi}^{2} / M^{2}\right)+\frac{A \xi M^{5}}{4 M_{\mathrm{pl}}^{2}}\left(\ln \frac{\bar{\phi}}{M}-c+1\right)\right] \frac{d \phi}{d \bar{\phi}}} d \phi$,

whereas $N_{\max }$ is also changed to

$$
N_{\max } \equiv \int_{\phi_{\mathrm{end}}}^{\phi=0} \frac{V_{E}}{V_{E}^{\prime}} d \phi
$$

The resultant $n_{s}$ in the model with a logarithmic form of nonminimal coupling to gravity is shown in Fig. 3. Left and right panels correspond to $\xi=10^{-16}$ and $10^{-8}$, respectively. Lines and regions in the figures represent the same meaning as those in Fig. 1. Similar to the case of the $\mathrm{CW}$ model with a linear term, we define

$$
\begin{gathered}
\tilde{C} \equiv\left(\frac{M_{\mathrm{pl}}}{M}\right)^{3} \frac{C}{A M^{3}}, \\
C \equiv \frac{c A \xi M^{5}}{4 M_{\mathrm{pl}}^{2}},
\end{gathered}
$$

so that this parametrization leads to the $-C \phi$ term in Eq. (14). One can approximate Eq. (14) as

$$
V_{E}(\bar{\phi}(\phi)) \simeq \begin{cases}\frac{A}{4} \bar{\phi}^{4}\left(\ln \frac{\bar{\phi}^{2}}{M^{2}}-\frac{1}{2}\right)-C \phi+V_{0} & \text { for } \quad|\ln (\bar{\phi} / M)| \ll c \\ \frac{A}{4} \bar{\phi}^{4}\left(\ln \frac{\bar{\phi}^{2}}{M^{2}}-\frac{1}{2}\right)+\frac{C \bar{\phi}}{c} \ln \frac{\bar{\phi}}{M}+V_{0} & \text { for } \quad|\ln (\bar{\phi} / M)| \gg c\end{cases}
$$

with the dimension-full parameter $C$ defined in Eq. (22). The condition $|\ln (\bar{\phi} / M)| \ll c$ corresponds to

$$
\mathcal{O}\left(10^{1-2}\right) \times \frac{\xi M_{\mathrm{pl}}}{4 \tilde{C}} \ll M,
$$

with the use of the parameter $\tilde{C}$ where a coefficient $\mathcal{O}\left(10^{1-2}\right)$ corresponds to $|\ln (\bar{\phi} / M)|$. In this region, the model gives a similar prediction to the $\mathrm{CW}$ model with a linear term. On the other hand, the term $\left(2 \xi M V_{0} \bar{\phi} / M_{\mathrm{pl}}^{2}\right) \ln (\bar{\phi} / M)$ in Eq. (14), which is the $(C \bar{\phi} / c) \ln (\bar{\phi} / M)$ term in Eq. (23), becomes effective compared to the linear term in the region of $M \ll \mathcal{O}\left(10^{1-2}\right) \times\left(\xi M_{\mathrm{pl}}\right) /(4 \tilde{C})$. Such regions appear around
$M \lesssim 10^{17} \mathrm{GeV}$ in the right panel of Fig. 3; that is, the logarithmic term of Eq. (13) becomes dominant at $M \lesssim 10^{17} \mathrm{GeV}$. We find that the model can have parameter space where constraints from cosmological observations can be satisfied when one takes $10^{-6} \lesssim \tilde{C} \lesssim 10^{-5}$ and $\xi \lesssim 10^{-8}$ for $40 \leq N_{*} \leq 55$ in the broad region of $M$. Interestingly, the effect of logarithmic contribution in nonminimal coupling can give larger $n_{s}$ in the region of $45 \lesssim N_{*} \lesssim 55$ compared to the model of fermion condensates. The additional contribution of $\xi$ in the denominator of Eq. (19) changes the value of $N_{*}$, but it does not change the magnitude of $\epsilon$, so the tensor-to-scalar ratio remains tiny. 

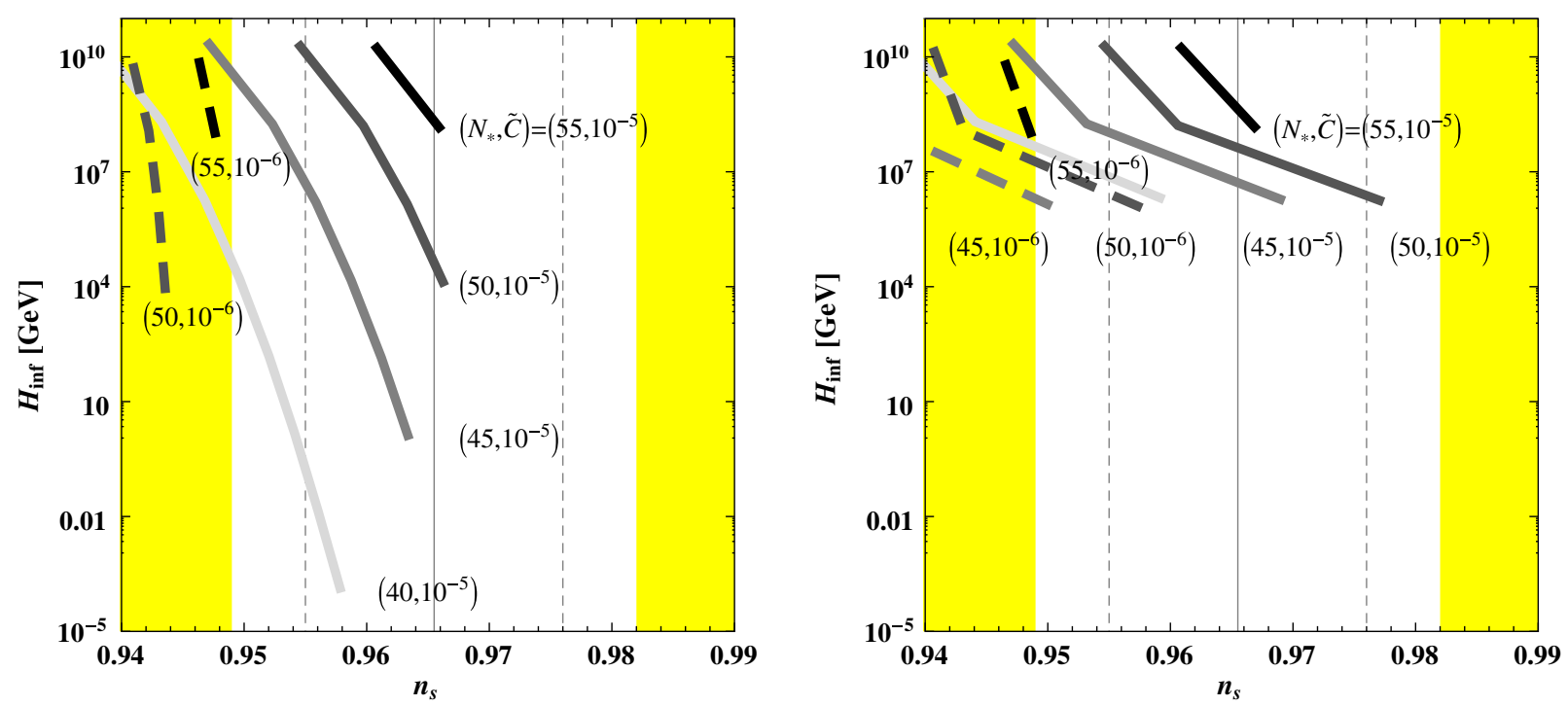

FIG. 4. $H_{\text {inf }}$ in the $\mathrm{CW}$ model with a logarithmic form of nonminimal coupling to gravity. Left and right panels correspond to $\xi=10^{-16}$ and $10^{-8}$, respectively. The meanings of curves and region in the figures are the same as those in Fig. 1.
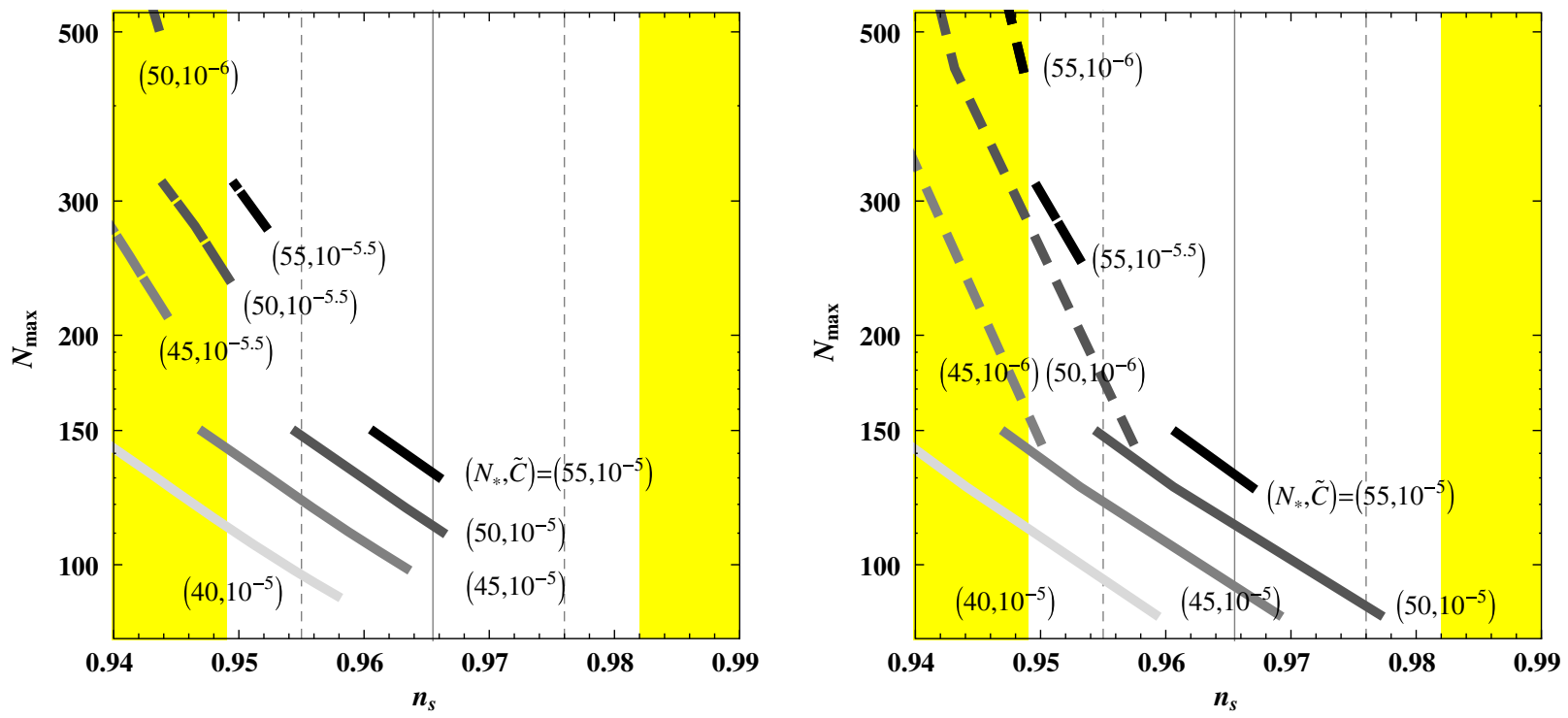

FIG. 5. $N_{\max }$ in the CW model with a logarithmic form of nonminimal coupling to gravity. Left and right panels correspond to $\xi=10^{-16}$ and $10^{-8}$, respectively. The meanings of curves and region in the figures are the same as those in the right panel of Fig. 2.

Figure 4 shows $H_{\text {inf }}$ as a function of $n_{s}$ for fixed $\xi, N_{*}$, and $\tilde{C}$. The curves and region in the figures are the same as those in Figs. 1 and 3. We find, in the left panel, that the possible inflation scale, $H_{\text {inf }} \gtrsim 10^{-4} \mathrm{GeV}, 1 \mathrm{GeV}, 10^{4} \mathrm{GeV}$, and $10^{8} \mathrm{GeV}$ for $N_{*}=40,45,50$, and 55 with an appropriate value of $\tilde{C}$ for a smaller $\xi$, is the same as that in the $\mathrm{CW}$ model with a linear term. For a larger $\xi$ shown in the right panel, we find that the change of the curve around $H_{\mathrm{inf}}=10^{8} \mathrm{GeV}$, where the logarithmic term becomes important, increases $n_{s}$ more significantly than the linear term.

We also evaluate $N_{\max }$ as shown in Fig. 5. The maximal value of the number of $e$-folds is $N_{\max } \simeq 110,140,300$, and
310 for $N_{*}=40,45,50$, and 55, respectively, with an appropriate value of $\tilde{C}$. The maximal value of $N_{*}$ is similar to that in the case of the $\mathrm{CW}$ model with a linear term.

\section{CONCLUSIONS}

The CW potential for an inflaton realizes a small-field inflation, but the current bound on $n_{s}$ from cosmological observation rules out the simplest small-field $\mathrm{CW}$ inflation model with smaller $M$. Thus, some modifications are necessary for such models to be consistent with cosmological observations. 
TABLE I. Summary of minimal values of $H_{\text {inf }}\left(\equiv H_{\mathrm{inf}}^{\min }\right)$ and the number of $e$-folds, $\left(H_{\mathrm{inf}}^{\min }, N_{\mathrm{max}}\right)$, for possible additional terms and $N_{*}$.

\begin{tabular}{lcccc}
\hline \hline Additional term $\backslash N_{*}$ & 40 & 45 & 50 & 55 \\
\hline Logarithmic & $\left(10^{-4} \mathrm{GeV}, 110\right)$ & $(1 \mathrm{GeV}, 140)$ & $\left(10^{4} \mathrm{GeV}, 300\right)$ & $\left(10^{8} \mathrm{GeV}, 310\right)$ \\
Linear & $\left(10^{-4} \mathrm{GeV}, 110\right)$ & $(1 \mathrm{GeV}, 140)$ & $\left(10^{4} \mathrm{GeV}, 240\right)$ & $\left(10^{8} \mathrm{GeV}, 310\right)$ \\
\hline \hline
\end{tabular}

An introduction of a linear term in the inflaton potential, which can be induced from fermion condensate, has been proposed to make the model realistic. In this work, first we have revisited this model with a linear term. In particular, we have investigated the inflation scale, that is, the Hubble scale during inflation $H_{\text {inf }}$, and maximal value of number of $e$-folds $N_{\max }$ in parameter space, where cosmological bounds are satisfied. These two quantities would be relevant to various cosmological scenarios or problems. The lowest possible inflation scale is $H_{\text {inf }} \gtrsim 10^{-4} \mathrm{GeV}, 1 \mathrm{GeV}, 10^{4} \mathrm{GeV}$, and $10^{8} \mathrm{GeV}$ for $N_{*}=40,45,50$, and 55, respectively, with $\tilde{C}=10^{-5}$, and the maximal value of the number of $e$-folds is $N_{\max } \simeq 110,140,240$, and 310 for $N_{*}=40,45,50$, and 55, respectively, with an appropriate value of $\tilde{C}$.

Next, we have proposed another possible realization of the small-scale CW inflation, where the linear and logarithmic term of nonminimal coupling is introduced. This type of model also includes a parameter space where the model becomes similar to the $\mathrm{CW}$ model with a linear term. Regarding a possible inflation scale, we find $H_{\text {inf }} \gtrsim 10^{-4} \mathrm{GeV}, 1 \mathrm{GeV}, 10^{4} \mathrm{GeV}$, and $10^{8} \mathrm{GeV}$ for

[1] A. A. Starobinsky, Phys. Lett. 91B, 99 (1980).

[2] K. Sato, Mon. Not. R. Astron. Soc. 195, 467 (1981).

[3] A. H. Guth, Phys. Rev. D 23, 347 (1981).

[4] V. F. Mukhanov and G. V. Chibisov, Pis'ma Zh. Eksp. Teor. Fiz. 33, 549 (1981) [JETP Lett. 33, 532 (1981)].

[5] S. W. Hawking, Phys. Lett. 115B, 295 (1982).

[6] A. A. Starobinsky, Phys. Lett. 117B, 175 (1982).

[7] A. H. Guth and S. Y. Pi, Phys. Rev. Lett. 49, 1110 (1982).

[8] A. D. Linde, Phys. Lett. 129B, 177 (1983).

[9] J. H. Kung and R. H. Brandenberger, Phys. Rev. D 42, 1008 (1990).

[10] R. D. Peccei and H. R. Quinn, Phys. Rev. Lett. 38, 1440 (1977).

[11] R. D. Peccei and H. R. Quinn, Phys. Rev. D 16, 1791 (1977).

[12] M. Axenides, R. H. Brandenberger, and M. S. Turner, Phys. Lett. 126B, 178 (1983).

[13] D. Seckel and M. S. Turner, Phys. Rev. D 32, 3178 (1985).

[14] A. D. Linde, Phys. Lett. 158B, 375 (1985).

[15] M. S. Turner and F. Wilczek, Phys. Rev. Lett. 66, 5 (1991).

[16] P. A. R. Ade et al. (Planck Collaboration), Astron. Astrophys. 594, A20 (2016).
$N_{*}=40,45,50$, and 55 , respectively, for $\tilde{C}=10^{-5}$ with small $\xi$. Thus, one can realize small $H_{\text {inf }}$ such as $10^{-4} \mathrm{GeV}$ at $M=10^{8} \mathrm{GeV}$. Lower bounds depend on the magnitude of $\xi$ as $H_{\text {inf }} \gtrsim 10^{-4}\left(10^{6}\right) \mathrm{GeV}$ for larger $\xi$ as $\xi=10^{-16}\left(10^{-8}\right)$. The model also gives the maximal value of the number of $e$-folds as $N_{\max } \simeq 110,140,300$, and 310 for $N_{*}=40,45,50$, and 55, respectively. The possible maximal value of $N_{*}$ is similar to that in the case of the $\mathrm{CW}$ model with a linear term.

In summary, the logarithmic nonminimal coupling can help make the small-scale CW inflation viable by increasing $n_{s}$. The nonminimal coupling can also realize a small inflation scale. In addition, motivated by the relaxion scenario, we have estimated the maximal number of $e$-folds, $N_{\max }$, which turns out to be $\mathcal{O}(100)$ and cannot be so enormous as required in relaxion models. The summary of possible additional terms to make the original $\mathrm{CW}$ model realistic is given in Table I.

\section{ACKNOWLEDGMENTS}

This work was supported by IBS under Project No. IBSR018-D1.
[17] I. Affleck and M. Dine, Nucl. Phys. B249, 361 (1985).

[18] M. Dine, L. Randall, and S. D. Thomas, Nucl. Phys. B458, 291 (1996).

[19] K. Enqvist and J. McDonald, Nucl. Phys. B538, 321 (1999).

[20] L. Roszkowski and O. Seto, Phys. Rev. Lett. 98, 161304 (2007).

[21] O. Seto and M. Yamaguchi, Phys. Rev. D 75, 123506 (2007).

[22] D. V. Nanopoulos, K. A. Olive, and M. Srednicki, Phys. Lett. 127B, 30 (1983).

[23] M. Y. Khlopov and A. D. Linde, Phys. Lett. 138B, 265 (1984).

[24] J. R. Ellis, J. E. Kim, and D. V. Nanopoulos, Phys. Lett. 145B, 181 (1984).

[25] M. Kawasaki, K. Kohri, T. Moroi, and A. Yotsuyanagi, Phys. Rev. D 78, 065011 (2008).

[26] R. H. Cyburt, J. Ellis, B. D. Fields, F. Luo, K. A. Olive, and V. C. Spanos, J. Cosmol. Astropart. Phys. 10 (2009) 021.

[27] P. W. Graham, D. E. Kaplan, and S. Rajendran, Phys. Rev. Lett. 115, 221801 (2015).

[28] T. Kobayashi, O. Seto, T. Shimomura, and Y. Urakawa, Mod. Phys. Lett. A 32, 1750142 (2017). 
[29] K. Choi and S. H. Im, J. High Energy Phys. 12 (2016) 093.

[30] B. Batell, G. F. Giudice, and M. McCullough, J. High Energy Phys. 12 (2015) 162.

[31] S. Di Chiara, K. Kannike, L. Marzola, A. Racioppi, M. Raidal, and C. Spethmann, Phys. Rev. D 93, 103527 (2016).

[32] J. L. Evans, T. Gherghetta, N. Nagata, and Z. Thomas, J. High Energy Phys. 09 (2016) 150.

[33] J. L. Evans, T. Gherghetta, N. Nagata, and M. Peloso, Phys. Rev. D 95, 115027 (2017).

[34] A. Hook and G. Marques-Tavares, J. High Energy Phys. 12 (2016) 101.

[35] T. Higaki, N. Takeda, and Y. Yamada, Phys. Rev. D 95, 015009 (2017).

[36] K. Choi, H. Kim, nd T. Sekiguchi, Phys. Rev. D 95, 075008 (2017).

[37] S. R. Coleman and E. J. Weinberg, Phys. Rev. D 7, 1888 (1973).

[38] A. D. Linde, Phys. Lett. 108B, 389 (1982).

[39] A. Albrecht and P. J. Steinhardt, Phys. Rev. Lett. 48, 1220 (1982).

[40] Q. Shafi and A. Vilenkin, Phys. Rev. Lett. 52, 691 (1984).

[41] G. Barenboim, E. J. Chun, and H. M. Lee, Phys. Lett. B 730, 81 (2014).

[42] N. Okada, V. N. Şenoğuz, and Q. Shafi, Turk. J. Phys. 40, 150 (2016).

[43] Q. Shafi and V. N. Senoguz, Phys. Rev. D 73, 127301 (2006).

[44] M. U. Rehman, Q. Shafi, and J. R. Wickman, Phys. Rev. D 78, 123516 (2008).
[45] K. Kannike, A. Racioppi, and M. Raidal, J. High Energy Phys. 01 (2016) 035.

[46] L. Marzola, A. Racioppi, M. Raidal, F. R. Urban, and H. Veermae, J. High Energy Phys. 03 (2016) 190.

[47] L. Marzola and A. Racioppi, J. Cosmol. Astropart. Phys. 10 (2016) 010.

[48] M. Artymowski and A. Racioppi, J. Cosmol. Astropart. Phys. 04 (2017) 007.

[49] S. Iso, K. Kohri, and K. Shimada, Phys. Rev. D 91, 044006 (2015).

[50] P. A. R. Ade et al. (BICEP2 and Keck Array Collaborations), Phys. Rev. Lett. 116, 031302 (2016).

[51] A. R. Liddle and D. H. Lyth, Phys. Rep. 231, 1 (1993).

[52] D. H. Lyth and A. Riotto, Phys. Rep. 314, 1 (1999).

[53] A. R. Liddle and S. M. Leach, Phys. Rev. D 68, 103503 (2003).

[54] A. De Simone, M. P. Hertzberg, and F. Wilczek, Phys. Lett. B 678, 1 (2009).

[55] I. L. Buchbinder and S. D. Odintsov, Yad. Fiz. 42, 1268 (1985); Classical Quantum Gravity 2, 721 (1985).

[56] E. Elizalde and S. D. Odintsov, Phys. Lett. B 303, 240 (1993); Russ. Phys. J. 37, 25 (1994).

[57] A. Salvio and A. Strumia, J. High Energy Phys. 06 (2014) 080.

[58] K. Kannike, G. Hutsi, L. Pizza, A. Racioppi, M. Raidal, A. Salvio, and A. Strumia, J. High Energy Phys. 05 (2015) 065.

[59] A. Salvio, Eur. Phys. J. C 77, 267 (2017).

[60] A. Karam, T. Pappas, and K. Tamvakis, Phys. Rev. D 96, 064036 (2017). 